\title{
On the Relationship between Pipe Dreams and Permutation Words
}

\author{
Cameron Marcott* \\ cam.marcott@gmail.com
}

Submitted: Nov 20, 2012; Accepted: Sep 11, 2013; Published: Sep 20, 2013

Mathematics Subject Classifications: 05A05, 06A07, 14M15

\begin{abstract}
Pipe dreams represent permutations pictorially as a series of crossing pipes. Recent applications of pipe dreams include the calculation of Schubert polynomials, fillings of moon polyominoes, and in the combinatorics of antidiagonal simplicial complexes. These applications associate pipe dreams to words of elementary symmetric transpositions via a canonical mapping. However, this canonical mapping is by no means the only way of mapping pipe dreams to permutation words. We define sensical mappings from pipe dreams to words and prove sensical mappings are in bijection with standard shifted tableaux of triangular shape. We characterize the set of pipe dreams associated to a given word (under any sensical map) using step ladder moves. These moves induce a partial order on the set of pipe dreams mapping to a given word, yielding a distributive lattice.
\end{abstract}

\section{Introduction}

RC-graphs were introduced by Fomin and Kirillov in [4] to aid in the calculation of Schubert polynomials. This work was further developed by Bergeron and Billey in [2]. Recently, Serrano and Stump in [11] and Rubey in [10] observed a correspondence between RC-graphs and certain 0-1-fillings of moon polyominoes. This relationship yielded a positivity result for Schubert polynomials. Miller and Sturmfels redubbed RC-graphs as pipe dreams and employed them to study the combinatorics of antidiagonal simplicial complexes in their book [8].

Most applications study pipe dreams as they relate to a particular permutation. This paper instead studies how pipe dreams relate to reduced words in the symmetric group. This study is motivated by two facts: (i) some formulations for computing Schubert polynomials with pipe dreams involve summing over reduced words, and (ii) that pipe

${ }^{*}$ Supported by the Kay Winger Blair Fund, St. Olaf College 
dreams look suspiciously similar to wiring diagrams, a pictorial representation of words in the symmetric group. We observe that there are multiple ways to map pipe dreams to words which preserve certain nice properties. We call these sensical mappings and show that sensical mappings are in bijection with standard shifted tableaux of triangular shape. We provide algorithms for finding which reduced words have pipe dreams mapping to them under a particular sensical map and show that all of the pipe dreams mapping to a given word are related by step ladder moves. Step ladder moves induce a partial order on the pipe dreams mapping to a given word, yielding a distributive lattice.

Throughout this paper $\sigma_{i}$ is the $i$-th elementary symmetric transposition. That is, $\sigma_{i}$ is the permutation in $S_{n}$ that swaps $i$ and $i+1$. Composition of transpositions is read in the typical right to left fashion (i.e. $\sigma_{2} \sigma_{1}=(3,1,2)$ in one line notation). A word is a string of elementary transpositions. A transposition in the string is called a letter. A word is reduced if it is a minimal length expression for the permutation obtained by composing its letters. All words considered in this paper are reduced unless otherwise noted.

\section{Pipe Dreams}

To build an $n \times n$ pipe dream draw an $n+1 \times n+1$ grid and enumerate it from 1 to $n$ down the left side and along the top (coordinates are given in the form (row, column)). Fill in

the internal squares with crossing pieces $t$ or elbow tiles $r$. Pipe dreams correspond to permutations by sending a number along the top to the number its pipe leads to on the left. Pipes are named after the number they originate at; the pipe starting at 3 is called pipe number 3. A pipe dream is called reduced if any pair of pipes cross at most once. All pipe dreams considered in this paper are reduced unless otherwise noted. Following convention, simplify drawings by only filling squares in the grid's upper left triangle (any crossing pieces outside of this triangle will either not affect the permutation or send a pipe from the top into the hinterlands below the grid).

Example 1. A pipe dream corresponding to the permutation $(2,3,1)$.

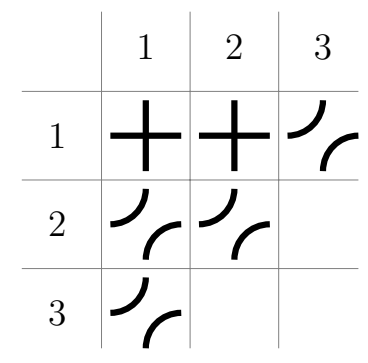

\section{Pipe Dreams to Words - Sensical Mappings}

A pipe dream with exactly one crossing tile at $(i, j)$ performs the elementary symmetric transposition $\sigma_{i+j-1}$. This observation motivates the definition of a sensical mapping. 
Definition 2. A mapping from pipe dreams to permutation words is sensical if a crossing of pipes $a$ and $b$ in square $(i, j)$ maps to the letter $\sigma_{i+j-1}$ performing the swap $(a, b)$.

A sensical mapping from pipe dreams to words is then an ordering on the squares in the pipe dream such that, no matter where the pipe dream's crossing tiles are located, the letters corresponding to the crossing tiles perform the correct swaps when written in the chosen order. One way to order the letters falls right out of the original definition of pipe dreams as RC-graphs and has been observed previously [2].

Definition 3. Given a reduced word $w=w_{1} w_{2} \cdots w_{m}$ (where the $w_{i}$ 's are elementary symmetric transpositions), a $w$-compatible sequence is a sequence of positive integers $w^{\prime}=$ $w_{1}^{\prime}, w_{2}^{\prime}, \cdots, w_{m}^{\prime}$ such that

- $w_{i}^{\prime} \leqslant w_{i+1}^{\prime}$.

- If $w_{i}=\sigma_{j}$, then $w_{i}^{\prime} \leqslant j$.

- If $w_{i}=\sigma_{j}$ and $w_{i+1}=\sigma_{k}$ where $k>j$, then $w_{i+1}^{\prime}>w_{i}^{\prime}$.

Following convention, compatible sequences are written in array with the word they are associated to in the form $\left(\begin{array}{llll}w_{1}, & w_{2}, & \cdots & w_{m} \\ w_{1}^{\prime}, & w_{2}^{\prime}, & \cdots & w_{m}^{\prime}\end{array}\right)$.

To build a pipe dream from a word $w$ and compatible sequence $w^{\prime}$, put crossing pieces in the squares $\left(w_{i}^{\prime}, w_{i}-w_{i}^{\prime}+1\right)$. To extract a word and compatible sequence from an $n \times n$ reduced pipe dream, examine squares working leftward across rows starting with $(1, n)$. When the end of one row is reached, examine squares working leftward across the next row down eventually ending with the square $(n, 1)$. When there is a crossing piece in square $(i, j)$, add the letter $\sigma_{i+j-1}$ to the word and the integer $j$ to the compatible sequence. Note, letters and numbers are added to the right end of both the word and sequence. So, the pipe dream in Example 1 maps to the compatible sequence $\left(\begin{array}{ll}3, & 2 \\ 1, & 1\end{array}\right)$. This bijection meets the requirements of a sensical map. Call this map from pipe dreams to words the canonical map. The canonical map is not however the only sensical map from pipe dreams to words.

Theorem 4. Sensical maps from $n \times n$ pipe dreams (reduced or otherwise) to words in $S_{n}$ are in bijection with standard shifted tableaux of triangular shape with largest part size $n-1$.

A shifted Young diagram is a Young diagram whose the $i$-th row has been shifted to the right by $i$ units. A standard shifted tableau with $k$ boxes is a shifted Young diagram with $k$ boxes filled with the integers 1 through $k$ such that the numbers in any row (column) increase when read left to right (top to bottom). 
Proof. The goal is to order the $\left(\begin{array}{l}n \\ 2\end{array}\right)$ filled squares in the pipe dream such that a crossing of pipes $a$ and $b$ in square $(i, j)$ corresponds to the letter $\sigma_{i+j-1}$ performing the swap $(a, b)$ for any possible arrangement of crossing pieces. To find orders that work in general, find orders that work on the $n \times n$ pipe dream that is totally saturated with crossing tiles. Since any other $n \times n$ pipe dream's crossing tiles are a subset of the crossing tiles in the saturated pipe dream, a good ordering on the saturated one will be good for all others.

In general, the only crossing tiles that are guaranteed to switch adjacent pipes are the top rightmost and right topmost crossing tiles (that is, the crossing tile which has no crossing tiles weakly below it and no crossing tiles in its row that are to its left and the crossing tile which has no crossing tiles weakly to its right and no crossing tiles in its column that are above it). These crossing tiles may be the same or different. Pick one of these two tiles and remove it. Swap the numbers of the pipes that crossed at the removed tile the top row. The reader can check that following numbers on the top to numbers on the left in the new figure, the final permutation will be preserved.

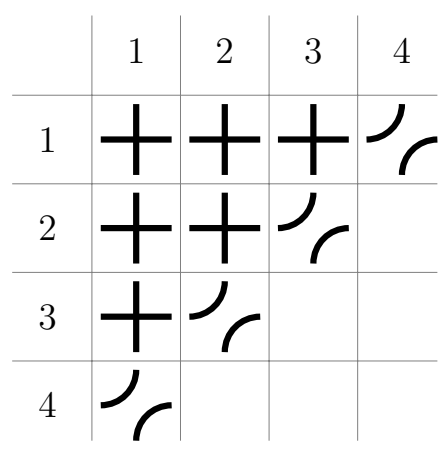

The saturated pipe dream

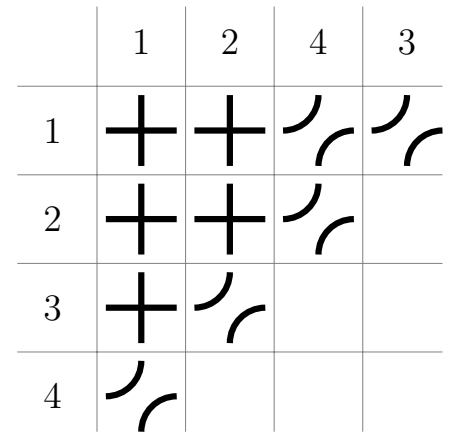

Remove a crossing and switch the labels

Continue picking either the top rightmost or right topmost crossing tile, removing it, and swapping the labels of the crossed pipes until the crossing free pipe dream is reached. The order the tiles were removed gives a sensical map from $n \times n$ pipe dreams to words. Because any sensical map must work for the saturated pipe dream in particular, all sensical maps can be constructed in this way.

Numbering squares in the order they were removed, digits must increase downward and to the left. In fact, if tiles are removed in an order given by a numbering of the squares that increases downward and to the left, the tile removed at any step will be either the right topmost or top rightmost tile and the ordering will give a sensical map. Ordering the boxes in a fashion that is not always increasing downward and to the left will at some point force a crossing tile that is not the right topmost or top rightmost to be removed. Then, the removed tile would not be guaranteed to switch two adjacent pipes and the map given by the ordering would not fulfill our criteria for a sensical mapping.

So, sensical maps are in bijection with leftward, downward increasing numberings of the boxes in the upper triangle. Reflecting the triangle and numbers gives a standard shifted tableau. Thus, sensical maps from $n \times n$ pipe dreams to words in $S_{n}$ are in bijection with standard shifted tableaux of triangular shape with largest part size $n-1$. 
Example 5. A Sensical Map.

\begin{tabular}{|l|l|l|}
\hline 4 & 2 & 1 \\
\cline { 1 - 2 } 5 & 3 & \multicolumn{1}{|c}{} \\
\cline { 1 - 2 } 6 & \multicolumn{2}{|c}{} \\
\cline { 1 - 1 } &
\end{tabular}

Note that the maps given in by this result are indeed different from the canonical map. For example, no pipe dream maps to the word $\sigma_{3} \sigma_{2} \sigma_{3} \sigma_{1}$ under the canonical map even though the following pipe dream maps to it under the map given in Example 5.

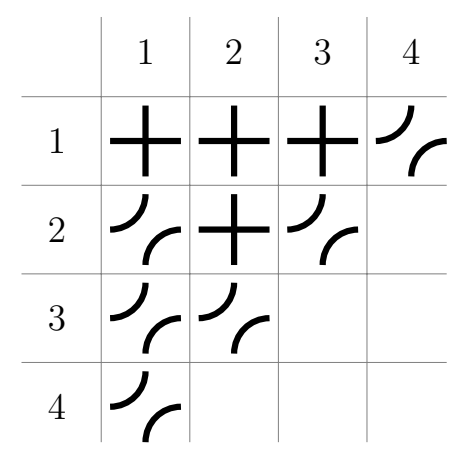

To see where this pipe dream maps to under the map of Example 5, simply examine the squares in the order dictated by the map. Whenever a crossing pieces occurs, write down the letter corresponding to that crossing's location adding new letters on the right. Indeed, the above pipe dream maps to $\sigma_{3} \sigma_{2} \sigma_{3} \sigma_{1}$. Under the canonical map, the above pipe dream would map to $\sigma_{3} \sigma_{2} \sigma_{1} \sigma_{3}$.

\section{Pipe Dreams Mapping to a Given Word}

Given a word in $S_{n}, w=w_{1} \cdots w_{m}$, when is there a pipe dream that maps to it under the canonical map? Under any sensical map? An easy test comes from the notion of compatible sequences. Break $w$ up into maximal length strictly decreasing (with regard to the letters' indices, $\sigma_{2}<\sigma_{3}$ ) subsequences. Build a sequence by associating a string of 1 's to the first decreasing subsequence, a string of 2's to the second and so on.

Lemma 6. Using the sequence constructed above, if any $w_{i}=\sigma_{j}$ has an associated value that is strictly greater than $j$, then the word does not have a compatible sequence and no pipe dream is associated with it. Otherwise, the resulting sequence is a compatible sequence which gives an associated pipe dream.

Proof. Assume that $w_{i}=\sigma_{j}$ and has an associated value that is strictly greater than $j$ in the sequence constructed above. Because of the requirement that adjacent letters with increasing indices in $w$ correspond to increasing values in a compatible sequence, all values 
in a compatible sequence for $w$ must be at least as large as the corresponding entry in the sequence constructed above. Then, the requirement that $w_{i}^{\prime} \leqslant j$ cannot be satisfied. The second result follows directly from the definition of a compatible sequence.

Note that the constructed sequence is the lexicographic minimum of all compatible sequences for $w$. By a mild abuse of language, call the pipe dream given by this sequence $w$ 's minimal pipe dream under the canonical map.

This test generalizes to sensical mappings. Take an ordering of tiles yielding a sensical map. Starting at $w_{1}$, for each $w_{i}=\sigma_{j}$, put a crossing piece in the smallest numbered box that corresponds to $\sigma_{j}$. Fill any smaller numbered boxes with elbow pieces and proceed to the next letter. Once $w_{k}$ has been reached, fill all remaining tiles with elbow pieces.

Lemma 7. If the above procedure fails to produce a pipe dream (if at any point there is no available square corresponding to $\sigma_{j}$ in which to put a crossing piece), then there are no pipe dreams corresponding to $w$ under the chosen map. Otherwise, the constructed pipe dream maps to the chosen word under the chosen map and is considered the minimal pipe dream to do so.

Proof. The proof is similar to Lemma 6.

Sensical mappings are not injective; three different pipe dreams map to $\sigma_{3}$ under any sensical map. How are the different pipe dreams mapping to a word under a given sensical map related? Previous literature shows that all pipe dreams of a given permutation are related by a series of chute and ladder moves [2], [8]; Rubey generalizes the notion of chute moves to obtain results on 0,1-fillings of moon polynominoes [10]; and Knutson, Miller and Sturmfels show pipe dreams of a given permutation can generate pipe dreams of smaller permutations via a process called mitosis [5], [7], [8]. So, the goal of finding mutation like relations between pipe dreams is very much in the spirit of previous work in the field.

Indeed there exists a series of moves relating pipe dreams associated to a given word under a given sensical map. In Section 5, we show that these moves give a decomposition of the chute poset of pipe dreams of a given permutation into disjoint distributive lattices. For the sake of having a concrete example, results are shown for the canonical map, then generalized to arbitrary sensical maps.

Definition 8. A step ladder move is a swap of the form

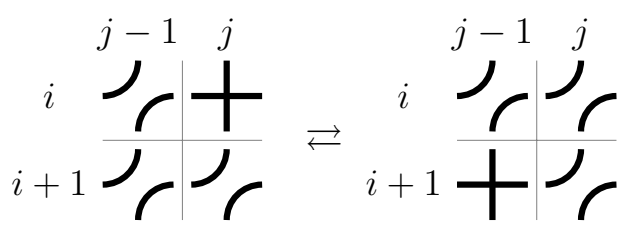

where there are no crossing pieces in row $i$ to the left of column $j$ and no crossing pieces in row $i+1$ to the right of column $j-1$. 
Theorem 9. Two pipe dreams map to the same word under the canonical map if and only if they are related by a series of step ladder moves.

Proof. Suppose that two pipe dreams are related by a step ladder move. Because any crossing piece slides only along the NE diagonal, the letters mapped to will be the same (in the figure in Definition 8, the image on the left has a crossing piece at $(i, j)$ which maps to $\sigma_{i+j-1}$ and the image on the right has a crossing piece at $(i+1, j-1)$ which also maps to $\left.\sigma_{i+j-1}\right)$. Since there are no crossing tiles to the left of column $j$ in row $i$ and no crossing tiles to the right of column $j-1$ in row $i+1$, the letters mapped to will be in the same order (this follows directly from the construction of the canonical map). So, two pipe dreams related by a step ladder move map to the same word under the canonical map.

Consider the pipe dream given by the word $w=w_{1} w_{2} \cdots w_{m}$ and the compatible sequence $w^{\prime}=\left(\begin{array}{cccc}w_{1}, & w_{2}, & \cdots & w_{m} \\ w_{1}^{\prime}, & w_{2}^{\prime}, & \cdots & w_{m}^{\prime}\end{array}\right)$. We show that $w^{\prime}$ 's minimal pipe dream under the canonical map is related to the given pipe dream by a series of downward moving step ladder moves. Then, any two pipe dreams mapping to $w$ are related to each other by a series of step ladder moves via w's minimal pipe dream. To this end, construct w's lexicographically minimal compatible sequence $w^{\prime \prime}=w_{1}^{\prime \prime}, w_{2}^{\prime \prime}, \cdots, w_{m}^{\prime \prime}$ using the method described in Lemma 6 . Transform $w^{\prime \prime}$ into $w^{\prime}$ by repeatedly increasing $w_{m}^{\prime \prime}$ by adding one until $w_{m}^{\prime \prime}=w_{m}^{\prime}$, then repeatedly increasing $w_{m-1}^{\prime \prime}$ by adding one until $w_{m-1}^{\prime \prime}=w_{m-1}^{\prime}$, and so on until $w_{1}^{\prime \prime}=w_{1}^{\prime}$. In pictorial terms, each time a digit is increased, a downward moving step ladder move is performed (increasing a digit slides a crossing piece down and to the left). Let $w_{i}=\sigma_{j}$ and $w_{i+1}=\sigma_{k}$. Due to the requirement that

$$
(k>j) \Rightarrow\left(w_{i+1}^{\prime}>w_{i}^{\prime}\right),
$$

and because we are working our way from $w_{m}^{\prime \prime}$ to $w_{1}^{\prime \prime}$, we conclude that

$$
\left(w_{i+1}^{\prime \prime}=w_{i}^{\prime \prime}\right) \Rightarrow(k<j) .
$$

In simpler pictorial language: if the row the moving tile originated has another crossing tile in it, that other tile must lie to the right of the moving tile. So, there are no tiles to the left of the moving tile in its starting row. Further, no tile can lie to the right of the moving tile in its destination row. If this were the case, there would exist some $i$ such that $w_{i}^{\prime}>w_{i+1}^{\prime}$, which cannot happen because $w^{\prime}$ is a compatible sequence.

So, any pipe dream mapping to $w$ is related to the pipe dream given by $w$ 's lexicographically minimal compatible sequence via a series of step ladder moves. Then, any two pipe dreams mapping to $w$ are related by a series of step ladder moves via the lexicographically minimal pipe dream. Thus, two pipe dreams map to the same word under the canonical map if and only if they are related by a series of step ladder moves.

The notion of a step ladder move can be easily generalized, giving similar results on when two pipe dreams corresponding to the same word under any sensical map are related. 
Definition 10. Choose a sensical map from pipe dreams to words and write it as in Example 5. A generalized step ladder move is a similar move to Definition 8 where there are no crossing tiles aside from the moving one in boxes numbered weakly larger than the box at $(i, j)$ and weakly less than the box at $(i+1, j-1)$. Note the squares filled with elbow joints in the picture are indeed necessarily elbow joint pieces as these squares must be numbered greater than the square at $(i, j)$ and less than the square at $(i+1, j-1)$.

Example 11. The following would be a step ladder move under the map from Example 5 even though it is not a step ladder move under the canonical map.

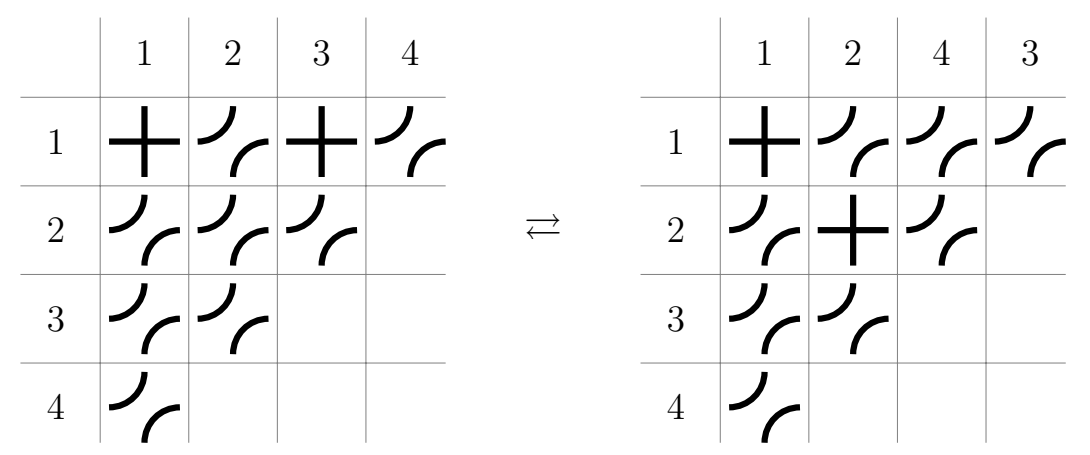

Theorem 12. Two pipe dreams map to the same word under a given map if and only if they are related by a series of generalized step ladder moves.

Proof. The proof is similar to Theorem 9.

\section{The Step Ladder Poset}

Bergeron and Billey noted that chute moves, beyond generating all pipe dreams associated to a given permutation, induce a partial ordering on pipe dreams of a given permutation. However, little study has gone into this poset. The chute poset for $(1,4,3,2)$ can be seen in Figure 1 (Bergeron and Billey give this poset as an example in their introduction to the topic, [2]).

The chute poset of pipe dreams associated to a given permutation is always graded. However, casual examination of Figure 1 shows that the chute poset is not in general a lattice, and not in general ranked. Defining a step ladder poset of pipe dreams associated to a given word yields a poset with these properties. Because step ladder moves are just special chute moves, one might be able to dig up some interesting properties of the chute poset of a given permutation by decomposing it into step ladder subposets given by the different reduced words for the permutation. However, that route is not one we pursue at this time.

To build the step ladder poset, consider the set of all pipe dreams mapping to a given word under a given sensical map. For pipe dreams $x$ and $y$, say $x \geqslant y$ if $y$ can be reached by performing a series of downward step ladder moves to $x$. For the following theorem, only the canonical mapping is considered. These results easily generalize to any sensical mapping. 


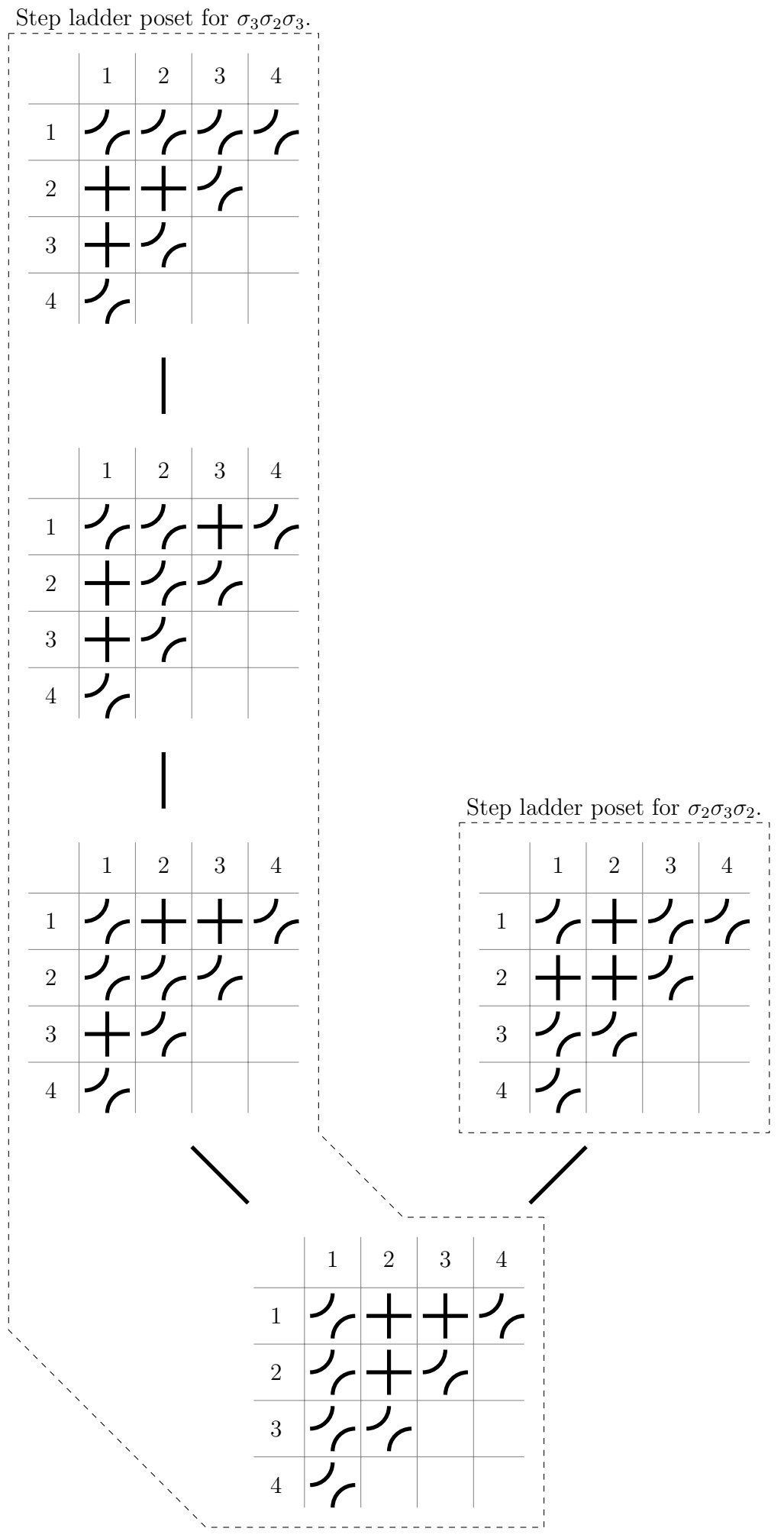

Figure 1: The chute poset for $(1,4,3,2)$ with decomposition into step ladder posets. 
Recall that a lattice is a poset in which any two elements have a supremum and infimum. Formally, in the case of the supremum, for any two elements $x$ and $y$ there is an element $x \vee y$ called the join of $x$ and $y$ such that $(x \vee y) \geqslant x$ and $y$ and for any $z$ such that $z \geqslant x$ and $y$, we have $(x \vee y) \leqslant z$. Similarly, in the case of the infimum, there is an element $x \wedge y$ called the meet of $x$ and $y$ such that $(x \wedge y) \leqslant x$ and $y$ and for any $z$ such that $z \leqslant x$ and $y$, we have $(x \wedge y) \geqslant z$.

A distributive lattice is a lattice in which meets and joins distribute. That is, for any elements $x, y$, and $z$ we have $x \vee(y \wedge z)=(x \vee y) \wedge(x \vee z)$ and $x \wedge(y \vee z)=(x \wedge y) \vee(x \wedge z)$.

Theorem 13. The step ladder poset is a distributive lattice.

For a word $w=w_{1}, w_{2}, \ldots, w_{m}$, let $x$ and $y$ be a pipe dreams associated to $w$ with compatible sequences $x^{\prime}=\left(\begin{array}{cccc}w_{1}, & w_{2}, & \cdots & w_{m} \\ x_{1}^{\prime}, & x_{2}^{\prime}, & \cdots & x_{m}^{\prime}\end{array}\right)$ and $y^{\prime}=\left(\begin{array}{cccc}w_{1}, & w_{2}, & \cdots & w_{m} \\ y_{1}^{\prime}, & y_{2}^{\prime}, & \cdots & y_{m}^{\prime}\end{array}\right)$ respectively. A lemma describing meets and joins in the step ladder poset helps prove the theorem.

Lemma 14. The join and meet of $x$ and $y, x \vee y$ and $x \wedge y$ respectively, are given respectively by the compatible sequences

$$
\begin{aligned}
& \left(\begin{array}{cccc}
w_{1}, & w_{2}, & \cdots & w_{m} \\
\max \left(x_{1}^{\prime}, y_{1}^{\prime}\right), & \max \left(x_{2}^{\prime}, y_{2}^{\prime}\right) & \cdots, & \max \left(x_{m}^{\prime}, y_{m}^{\prime}\right)
\end{array}\right) \\
& \text { and } \\
& \left(\begin{array}{cccc}
w_{1}, & w_{2}, & \cdots & w_{m} \\
\min \left(x_{1}^{\prime}, y_{1}^{\prime}\right), & \min \left(x_{2}^{\prime}, y_{2}^{\prime}\right) & \cdots, & \min \left(x_{m}^{\prime}, y_{m}^{\prime}\right)
\end{array}\right) .
\end{aligned}
$$

Proof. We give the proof for joins; the proof for meets is similar. Call the sequence for the proposed join $M^{\prime}=\left(\begin{array}{cccc}w_{1}, & w_{2}, & \cdots & w_{m} \\ M_{1}^{\prime}, & M_{2}^{\prime} & \cdots & M_{m}^{\prime}\end{array}\right)$, where $M_{i}^{\prime}=\max \left(x_{i}^{\prime}, y_{i}^{\prime}\right)$. $\operatorname{Begin}$ by checking that $M^{\prime}$ is a compatible sequence for $w$. Suppose for some $i$ that $x_{i}^{\prime} \geqslant y_{i}^{\prime}$, $w_{i}=\sigma_{j}$, and $w_{i+1}=\sigma_{k}$. Then, $M_{i}^{\prime} \leqslant M_{i+1}^{\prime}$ because $x_{i}^{\prime} \leqslant x_{i+1}^{\prime} . M_{i}^{\prime} \leqslant j$ because $x_{i}^{\prime}, y_{i}^{\prime} \leqslant j$. If $k>j$, then $x_{i}^{\prime}<x_{i+1}^{\prime}$ and thus $M_{i}^{\prime}<M_{i+1}^{\prime}$. So, all the necessary conditions are met and $M^{\prime}$ is a compatible sequence for $w$.

This pipe dream is indeed greater than both $x$ and $y$ : From $x_{1}^{\prime}, x_{2}^{\prime}, \ldots, x_{m}^{\prime}$, increase $x_{m}^{\prime}$ by repeatedly adding 1 until $x_{m}^{\prime}=M_{m}^{\prime}$. Then, increase $x_{m-1}^{\prime}$ by repeatedly adding 1 until $x_{m-1}=M_{m-1}^{\prime}$ and so on until $x^{\prime}=M^{\prime}$. By the same reasoning used in the proof of Theorem 9, each increase corresponds to a downward moving step ladder move. So, the proposed join is indeed greater than $x$. Likewise, the proposed join is greater than $y$.

It remains to be shown that any other pipe dream $z \geqslant x, y$, given by compatible sequence $z^{\prime}=\left(\begin{array}{cccc}w_{1}, & w_{2}, & \cdots & w_{m} \\ z_{1}^{\prime}, & z_{2}^{\prime} & \cdots & z_{m}^{\prime}\end{array}\right)$, must be greater than or equal to the proposed join. Any digit $z_{i}^{\prime}$ must be greater than $M_{i}^{\prime}$. Were this not the case, one of $x_{i}^{\prime}$ or $y_{i}^{\prime}$ would need to be decreased in order to reach $z_{i}^{\prime}$. Such a decrease would correspond either an upward moving step ladder move or a move that isn't a step ladder move at all. If 
$z_{i}^{\prime}>M_{i}^{\prime}$, then digit increasing motions can be performed between $M^{\prime}$ and $z^{\prime}$. Then, $z$ is strictly greater than the proposed join. So, $x \vee y$ is indeed given by the compatible sequence $M^{\prime}$. A similar argument shows that $x \wedge y$ is given by the compatible sequence

$$
\left(\begin{array}{cccc}
w_{1}, & w_{2}, & \cdots & w_{m} \\
\min \left(x_{1}, y_{1}\right), & \min \left(x_{2}, y_{2}\right), & \cdots & \min \left(x_{m}, y_{m}\right)
\end{array}\right) .
$$

Proof of Theorem 13. From Lemma 14, the step ladder poset has well defined meets and joins and is thus a lattice. It remains to be shown that meets and joins distribute. Let $z$

given by the compatible sequence $\left(\begin{array}{cccc}w_{1}, & w_{2}, & \cdots & w_{m} \\ z_{1}^{\prime}, & z_{2}^{\prime} & \cdots & z_{m}^{\prime}\end{array}\right)$ be any pipe dream. Then, the compatible sequence for the pipe dream $x \wedge(y \vee z)$ is

$$
\left(\begin{array}{cccc}
w_{1}, & w_{2}, & \cdots & w_{m} \\
\max \left(x_{1}, \min \left(y_{1}, z_{1}\right)\right), & \max \left(x_{1}, \min \left(y_{1}, z_{1}\right)\right), & \cdots & \max \left(x_{m}, \min \left(y_{m}, z_{m}\right)\right)
\end{array}\right) .
$$

But, for any $i$, the identity

$$
\max \left(x_{i}, \min \left(y_{i}, z_{i}\right)\right)=\min \left(\max \left(x_{i}, y_{i}\right), \max \left(x_{i}, z_{i}\right)\right)
$$

holds. So, $x \wedge(y \vee z)=(x \wedge y) \vee(x \wedge z)$ and the meet distributes. The join distributes similarly. Thus, the step ladder poset is a distributive lattice.

Since the step ladder poset is a distributive lattice, it is also modular, ranked, and graded.

\subsection{Posets Whose Order Ideals are Step Ladder Posets}

By the fundamental theorem of finite distributive lattices, a unique poset $P$ exists such that the step ladder poset is isomorphic to $J(P)$ (the poset of order ideals of $P$ with partial ordering given by inclusion, notation from Stanley, [12]). We end our investigation by showing how to find $P$ from any word. For the sake of brevity, only the canonical mapping is considered in this section. The construction easily generalizes to any sensical mapping.

All that is necessary to find $P$ is to find the nonzero join irreducible elements in the step ladder poset ( $P$ is isomorphic to the induced subposet given by these elements). The join irreducible elements are those that cover exactly one other element. If an element covers two others, it is clearly not join irreducible. If there is an element $z$ covering exactly one other element take a $x$ and a $y$ such that $z \geqslant x, y$. The the element covered by $z$ is also greater than or equal to both $x$ and $y$. So, $x \vee y \neq z$ and $z$ is join irreducible.

One pipe dream covers another if the first pipe dream can be transformed into the second by a single upward moving step ladder move. An upward moving step ladder move corresponds to decreasing exactly one digit of the pipe dream's associated compatible sequence by one such that the new sequence is also compatible. We give an algorithm for finding a pipe dream's join irreducible elements given the word and compatible sequence determining the pipe dream. 
Algorithm 15. If $w=w_{1}, w_{2}, \ldots, w_{m}$ is a reduced word and

$$
w^{\prime}=\left(\begin{array}{cccc}
w_{1}, & w_{2}, & \cdots & w_{m} \\
w_{1}^{\prime}, & w_{2}^{\prime}, & \cdots & w_{m}^{\prime}
\end{array}\right)
$$

a compatible sequence, then the pipe dream determined by $w^{\prime}$ is join irreducible if and only if there exists some $i$ such that:

- If $i \neq 1$, then $w_{1}^{\prime}=1$.

- For $h \neq i$, if $w_{h}=\sigma_{j}$ and $w_{h-1}=\sigma_{k}$ where $j<k$, then $w_{h}^{\prime}=w_{h-1}^{\prime}$.

- For $h \neq i$, if $w_{h}=\sigma_{j}$ and $w_{h-1}=\sigma_{k}$ where $j>k$, then $w_{h}^{\prime}=w_{h-1}^{\prime}+1$.

If $w^{\prime}$ meets these conditions, only the digit $w_{i}^{\prime}$ may be decreased. So, the pipe dream covers exactly one other pipe dream and is thus join irreducible. Picking what digit of the compatible sequence is to be decreased and what that digit is determines the rest of the sequence uniquely under the above conditions. If the uniquely determined sequence is a compatible sequence, the result is join irreducible. If something breaks in determining the sequence, there is no pipe dream which covers a single other pipe dream by decreasing the chosen digit of the compatible sequence by one. Then, checking each digit $w_{i}^{\prime}$ from 2 up to $j$ where $w_{i}=\sigma_{j}$ in this fashion will yield all join irreducible pipe dreams.

These results are just the tip of the iceberg of what might be done with the step ladder poset. I freely admit to not being an expert in the field of posets and encourage those who are to explore other properties of the step ladder poset and also the chute poset's decomposition into step ladder subposets.

\section{Acknowledgments}

I'd like to thank Kaisa Tiapale for the guidance she's given me in this project and Saint Olaf College for the generous support they provided me, in particular the Kay WingerBlair Fund for financial support. Thanks to James Lodovic, Ezra Miller, and Victor Reiner for editorial advice.

\section{References}

[1] Jason Bandlow. An elementary proof of the hook formula. Electronic Journal of Combinatorics, 15:R45, 2008. http://www.combinatorics.org/ojs/index.php/ eljc/article/view/v15i1r45.

[2] Nantel Bergeron and Sarah Billey. Rc-graphs and schubert polynomials. Experimental Mathematics, 2:257-269, 1993. http://projecteuclid.org/euclid.em/ 1048516036. 
[3] Michael Davis. The Geometry and Topology of Coxeter Groups, volume 32 of London Math. Society Monograph Series. Princeton University Press, Princeton, 2007. http: //www.math.osu.edu/ davis.12/davisbook.pdf.

[4] Sergey Fomin and Anatol Kirillov. The Yang-Baxter equation, symmetric functions, and schubert polynomials. Discrete Mathematics, 153:123-143, 1996. http://www. sciencedirect.com/science/article/pii/0012365X9500132G.

[5] Allen Knutson and Ezra Miller. The Gröbner geometry of Schubert polynomials. Annals of Mathematics, 161:1245-1318, 2005. http://annals.math.princeton. edu/2005/161-3/p04.

[6] C. Krattenthaler. Bijective proofs for the hook formulas for the number of standard tableaux, ordinary and shifted. Electronic Journal of Combinatorics, 2:R13, 1995. http://www. combinatorics .org/ojs/index.php/eljc/article/view/v2i1r13.

[7] Ezra Miller. Mitosis recursion for coefficients of Schubert polynomials. Journal of Combinatorial Theory, Series A, 103:223-235, 2003. http://www. sciencedirect. com/science/article/pii/S0097316503000207.

[8] Ezra Miller and Bernd Sturmfels. Combinatorial Commutative Algebra, volume 227 of Graduate Texts in Math. Springer-Verlag, New York, 2005.

[9] The On-Line Encyclopedia of Integer Sequences, http://oeis.org, 2012.

[10] Martin Rubey. Maximal 0-1-fillings of moon polyominoes with restricted chain lengths and rc-graphs. DMTCS Proceedings, 0:835-848, 2011. http://www.dmtcs . org/dmtcs-ojs/index.php/proceedings/article/view/dmA00173/3649.

[11] Luis Serrano and Christian Stump. Maximal fillings of moon polyominoes, simplicial complexes, and Schubert polynomials. Electronic Journal of Combinatorics, 19:P16, 2012. http://www.combinatorics.org/ojs/index.php/eljc/article/ view/v19i1p16.

[12] Richard Stanley. Enumerative Combinatorics, Vol. 1, volume 49 of Cambridge Studies in Advanced Math. Cambridge University Press, Cambridge, 2012. http: //www-math.mit.edu/ ${ }^{\text {rstan/ec/ec1.pdf }}$ 